Reprod. Nutr. Dévelop., 1985, 25 (1 B), 295-301.

\title{
Influence d'un régime hyperlipidique sur la lipogenèse, la mise en réserve et l'utilisation des lipides chez la ratte gestante
}

\author{
Odette CHAMPIGNY, Yvonne HITIER
}

avec la collaboration technique de Claude KOHL et S. RAIMBAULT

Centre de Recherches sur la Nutrition, C.N.R.S., 9, rue Jules-Hetzel, 92190 Meudon-Bellevue. France.

Summary. Effect of an hyperlipidic diet on lipogenesis and on the storage and mobilization of lipids in the pregnant rat.

The aim of the present investigation was to determine the amount of fat accumulated and mobilized during pregnancy in rats fed either an hyperglucidic or an hyperlipidic diet.

The feeding of an hyperlipidic diet increased the liver lipid content and did not modify the composition of the mammary gland.

Fat was deposited till day 19 of pregnancy; after that date the fat was mobilized. The increase and decrease of fat stores were independent of the diet consumed. Both were correlated with body changes so that the same linear relationship between the lipid content of the carcass and maternal body weight was observed at all stages of pregnancy.

\section{Introduction.}

En dépit des nombreux travaux consacrés aux variations de la composition corporelle de la femelle au cours de la gestation (Spray, 1950 ; Beaton et al., 1954 ; Salmon-Legagneur et Jacquot, 1961 ; Heap et Lodge, 1967 ; Knopp et al., 1973 et 1975 ; Remesar et al., 1981 ; Aitchison, Clegg et Vernon, 1982 ; Steingrimsdottir, Greenwood et Brasel, 1980 ; Naismith, Richardson et Pritchard, 1982) l'accord n'est pas encore fait sur l'importance et la chronologie de la constitution des réserves tant lipidiques que protéiques effectuées par la gestante.

Les conditions nutritionnelles imposées pendant la gestation jouent un rôle dans l'élaboration de réserves par la femelle et conditionnent sa production laitière ultérieure comme l'ont montré des travaux réalisés chez le Porc et chez le Rat (Perisse et Salmon-Legagneur, 1960 ; Salmon-Legagneur et al., 1960 ; Champigny, 1962). Les modulations nutritionnelles choisies ont été le plus souvent la limitation globale de nourriture ou la réduction spécifique de l'apport protéique : on s'est moins attaché à l'influence de la nature de l'énergie consommée. 
Dans un précédent travail $\left(^{*}\right)$ nous avons montré que la chronologie de I'induction des enzymes de la lipogenèse et celle de la synthèse réelle de lipides étaient effectivement modulées par la nature de l'énergie consommée. C'est ainsi que, comparée à celle d'un régime hyperglucidique, la consommation d'un régime hyperlipidique réprime l'activité lipogénétique en début de gestation. En revanche, l'excès de lipides alimentaires est sans effet durant la deuxième partie de la gestation où la lipogenèse est stimulée quel que soit le type de nutriment énergétique consommé.

Nous présentons ici les répercussions de la consommation de ces 2 types de régimes sur l'élaboration des réserves lipidiques et le développement de la glande mammaire au cours de la gestation.

\section{Matériel et méthodes.}

Des rattes Sprague-Dawley pesant 220 à $250 \mathrm{~g}$ en début d'expérience ont été utilisées. Deux régimes ont été choisis :

- un régime G (62\% de calories glucidiques, $12 \%$ de calories lipidiques) ;

- un régime hyperlipidique $L$ (19 \% de calories glucidiques, $55 \%$ de calories lipidiques).

Ces régimes ont été distribués ad libitum à partir du jour de l'accouplement, mis en évidence par la présence de spermatozoïdes dans le frottis vaginal.

Les rattes ont été sacrifiées après $12,18,20$ et 21 jours de gestation ; des femelles témoins non gravides ont été sacrifiées parallèlement après un temps comparable de consommation de régime. Foie et glande mammaire ont été prélevés rapidement et conservés à $-20^{\circ} \mathrm{C}$, ainsi que la carcasse éviscérée, jusqu'au dosage des lipides par gravimétrie après extraction par la méthode de Folch et al., 1956.

\section{Résultats.}

Les rattes n'ont pas totalement ajusté leur niveau d'ingestion au pouvoir calorique du régime, et les ingérés des rattes $L$ ont toujours été légèrement supérieurs à ceux des rattes $\mathrm{G}$.

TABLEAU 1

Chronologie de l'ingestion (Kcal/jour).

\begin{tabular}{lccccc}
\hline & Témoins & 12 jours & 18 jours & 20 jours & 21 jours \\
\cline { 2 - 6 } Lot $G$ & 64 & 80 & 87 & 87 & 75 \\
Lot $L$ & 67 & 88 & 108 & 99 & 89 \\
\hline
\end{tabular}

$(*)$ Soumis pour publication. 
L'ingestion du régime $L$ n'a pas modifié l'évolution pondérale du foie au cours de la gestation, mais a entraîné une légère augmentation de sa teneur, donc de son contenu en lipides $(P<0,05)$, comme en témoigne le tableau 2 .

Ni l'évolution pondérale de la glande mammaire, ni sa teneur en lipides, $n$ 'ont été affectées par le régime hyperlipidique.

L'évolution des lipides corporels au cours de la gestation est représentée dans la figure 1, parallèlement à celle du poids vif de la mère " vide " (c.-à-d. sans

TABLEAU 2

Poids et teneur en lipides du foie et de la glande mammaire au cours de la gestation.

\begin{tabular}{|c|c|c|c|c|c|}
\hline \multirow{2}{*}{ Régime } & \multirow{2}{*}{ Lot } & \multicolumn{2}{|c|}{ Foie } & \multicolumn{2}{|c|}{ Glande mammaire } \\
\hline & & $\begin{array}{l}\text { Poids } \\
\text { (g) }\end{array}$ & $\begin{array}{l}\text { Lipides } \\
\text { (mg/g) }\end{array}$ & $\begin{array}{l}\text { Poids } \\
\text { (g) }\end{array}$ & $\begin{array}{l}\text { Lipides } \\
\text { (mg/g) }\end{array}$ \\
\hline G & $\begin{array}{c}\text { Non gestantes } \\
12 \text { jours } \\
18 \text { jours } \\
20 \text { jours } \\
21 \text { jours }\end{array}$ & $\begin{array}{r}8,45 \pm 0,41 \\
12,50 \pm 0,38 \\
13,28 \pm 0,43 \\
14,52 \pm 0,31 \\
12,04 \pm 0,65\end{array}$ & $\begin{array}{l}38,6 \pm 1,7 \\
35,5 \pm 1,3 \\
35,7 \pm 3,0 \\
31,4 \pm 1,7 \\
35,3 \pm 2,7\end{array}$ & $\begin{array}{l}1,48 \pm 0,22 \\
3,29 \pm 0,26 \\
3,47 \pm 0,37 \\
3,97 \pm 0,23 \\
4,75 \pm 0,32\end{array}$ & $\begin{array}{l}480 \pm 25 \\
411 \pm 23 \\
372 \pm 21 \\
404 \pm 21 \\
409 \pm 26\end{array}$ \\
\hline L & $\begin{array}{c}\text { Non gestantes } \\
12 \text { jours } \\
18 \text { jours } \\
20 \text { jours } \\
21 \text { jours }\end{array}$ & $\begin{array}{r}8,29 \pm 0,41 \\
11,93 \pm 0,60 \\
14,16 \pm 0,23 \\
13,95 \pm 0,60 \\
12,15 \pm 0,45\end{array}$ & $\begin{array}{l}44,7 \pm 2,2 \\
51,5 \pm 5,4 \\
39,7 \pm 1,8 \\
43,3 \pm 1,3 \\
44,4 \pm 2,5\end{array}$ & $\begin{array}{l}1,70 \pm 0,16 \\
3,29 \pm 0,23 \\
4,73 \pm 0,21 \\
4,28 \pm 0,25 \\
5,36 \pm 0,27\end{array}$ & $\begin{array}{l}473 \pm 13 \\
475 \pm 39 \\
485 \pm 17 \\
455 \pm 42 \\
378 \pm 22\end{array}$ \\
\hline
\end{tabular}
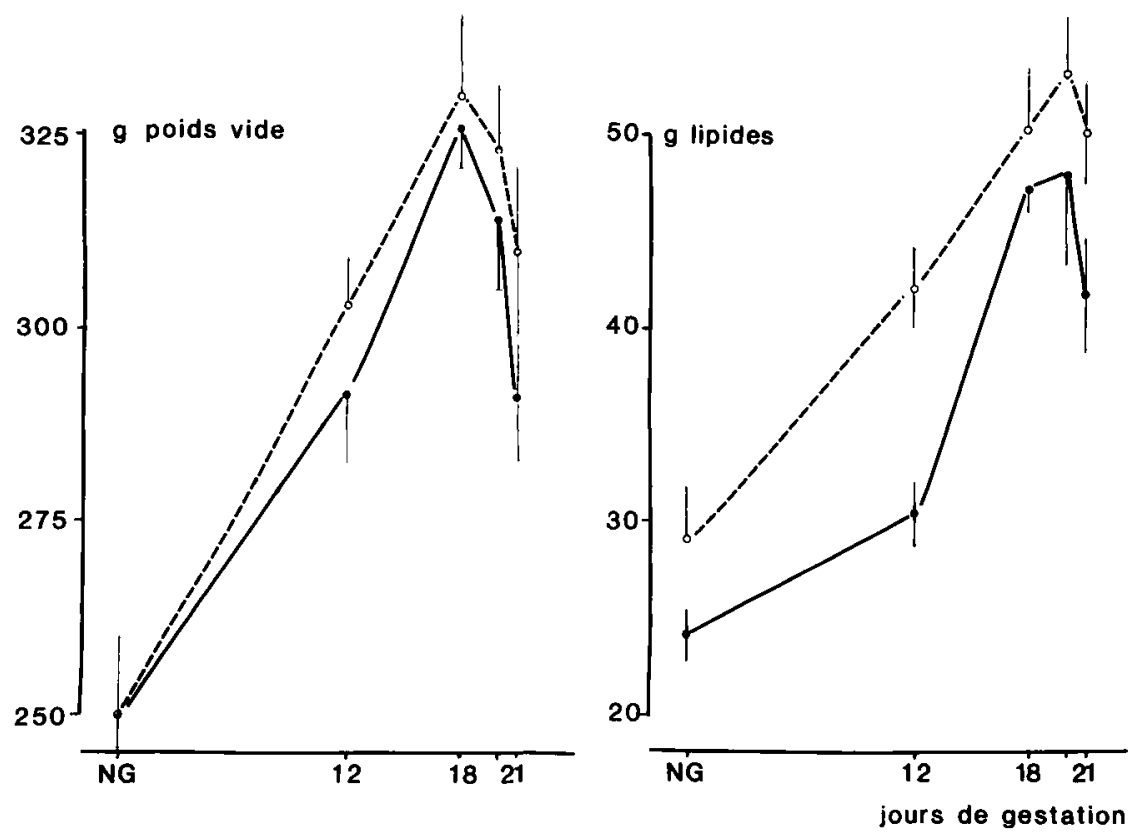

FIG. 1. - Evolution du poids vif de la ratte vide et des lipides corporels au cours de la gestation. régime $\mathrm{L}$ : $\mathrm{O}--$; régime $\mathrm{G}$ : 
utérus). La seule différence apportée par la consommation du régime $\mathrm{L}$ se situe dans la chronologie de la constitution des réserves lipidiques : elle est plus précoce qu'avec le régime $G$, et les deux lots diffèrent significativement à 12 jours de gestation. Le dépôt de lipides corporels se poursuit ensuite jusqu'à 18 jours et il est alors plus rapide dans le lot $G$, si bien qu'à 18 jours de gestation on ne note plus de différence entre les deux lots. Au-delà de cette date on observe une chute de poids vif et une baisse des lipides, analogues dans les deux lots.

Les lipides corporels exprimés en fonction du poids vif " vide " s'alignent sur une même droite (fig. 2) quels que soient l'âge de gestation ou le régime consommé. Cette droite a pour équation $Y=0,329 X-56,9$, où $Y$ représente le poids de lipides corporels et $X$ le poids vif.

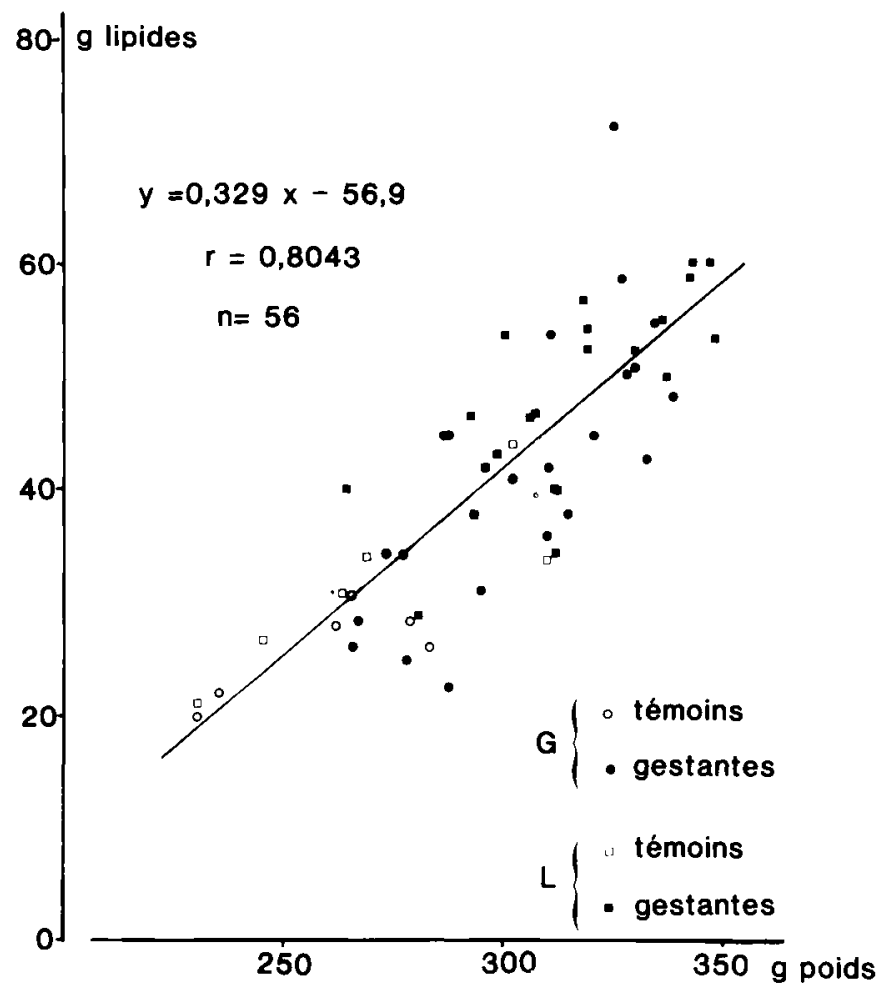

FIG. 2. - Relation entre lipides corpore/s et poids "vide " des rattes.

\section{Discussion.}

Si les auteurs sont d'accord pour constater en l'absence de restriction alimentaire une augmentation des lipides corporels au cours de la gestation, les opinions divergent en ce qui concerne l'importance, la durée et la signification de cette mise en réserve. A la suite de Spray (1950) et de Beaton et al. (1954) de 
nombreux auteurs divisent la gestation de la ratte en deux phases : une phase de lipogenèse pendant les deux premières semaines, une phase de lipolyse la $3^{e}$ semaine (Knopp et al., 1973 ; Remesar et al., 1981). D'autres situent cette phase de catabolisme lipidique plus tardivement, dans les derniers jours avant la parturition (Salmon-Legagneur et Jacquot, 1961 ; Knopp, Boroush et O'Sullivan, 1975 ; Flint et al., 1979 ; Humphrey et al., 1980 ; Aitchison, Clegg et Vernon, 1982). Nos résultats sont en accord avec ces derniers : I'accroissement des lipides corporels se poursuit bien au-delà du $14^{\mathrm{e}}$ jour de gestation. Après cette date la forte poussée de croissance fœtale n'empêche pas la prise de poids proprement maternelle, à condition que les conditions nutritionnelles soient convenables. Cette concomitance des croissances fœtale et maternelle est en effet rendue possible par l'augmentation de l'hyperphagie maternelle qu'on observe à cette période (Hitier et al., 1982). Après 18 jours de gestation l'appétit de la femelle diminue et elle fait alors appel à ses réserves corporelles, tant protéiques que lipidiques, pour couvrir les besoins fœtaux qui ne cessent d'augmenter.

L'importance des réserves lipidiques dépend de l'importance de la prise de poids, elle-même fonction du niveau d'ingestion (Childs et al., 1981 ; Herrera et Knopp, 1972). Cela ressort bien du travail de Naismith et al. (1982), où le gain de lipides des rattes gestantes par rapport aux témoins a varié d'une expérience à l'autre (46\% contre $92 \%$ ) parallèlement à une moindre augmentation du niveau d'ingestion $(17 \%$ au lieu de $23 \%)$. Cela est confirmé par la répartition linéaire des lipides corporels en fonction du poids vif que nous avons mise en évidence dans le présent travail. La pente de la droite, de l'ordre de $30 \%$, représente la part des lipides dans la variation de poids vif, qu'elle soit positive (avant 18 jours) ou négative (juste avant la mise-bas). Les rattes $L$, dont l'ingéré est supérieur à celui des rattes $\mathrm{G}$, ont seulement parcouru cette droite plus rapidement, sans faire des réserves lipidiques spécifiques plus élevées, pour un même poids vif, que les rattes $\mathrm{G}$. Nous ne retrouvons pas ici les résultats de Steingrimsdottir, Greenwood et Brasel (1980) qui, avec des rattes Osborne-Mendel, constatent que gestation et régime hyperlipidique ont des effets additifs et entraînent des dépôts exagérés de lipides corporels. Nous ne souscrivons pas non plus à la conclusion de Naismith, Richardson et Pritchard (1982) affirmant que les lipides sont stockés tout au long de la gestation et qu'il n'y a pas de perte lipidique au cours de la $3^{e}$ semaine. Si la quantité de lipides corporels à 21 jours est plus élevée qu'à 14 jours, ce que nous constatons également, cela ne veut pas dire que le dépôt de lipides ait été continu, sans mobilisation pendant toute cette période. L'existence d'une phase de lipolyse prépartum explique d'ailleurs l'augmentation des acides gras libres plasmatiques constatée par de nombreux auteurs (Knopp, Boroush et O'Sullivan, 1975 ; Wasfi, Weinstein et Heimberg, 1980).

L'évolution de la glande mammaire n'est pas modifiée par l'ingestion du régime $L$ et la préparation à la lactation est donc peu affectée par la consommation d'un régime riche en lipides.

En conclusion la nature du régime consommé (hyperglucidique ou hyperlipidique), si elle entraîne des modifications au niveau de la chronologie de la lipogenèse hépatique, ne modifie pas significativement la constitution des réserves lipi- 
diques au cours de la gestation, ni leur utilisation durant les derniers jours avant la parturition.

$10^{e}$ Réunion du groupe Développement I.N.R.A., Rennes, 9-10 mai 1984.

Remerciements. - Nous remercions G. Bourdel pour ses conseils et encouragements, et S. Finel pour le soin apporté à la réalisation du manuscrit.

\section{Références}

AITCHISON R. E. D., CLEGG R. A., VERNON R. G., 1982. Lipolysis in rat adipocytes during pregnancy and lactation. The response to noradrenaline. Biochem. J., 202, 243-247.

BEATON G. H., BEARE J., RYU M. H., Mc HENRY E. W., 1954. Protein metabolism in the pregnant rat $J$. Nutr., 54, 291-304.

CHAMPIGNY $0 ., 1962$. Influence d'une double restriction énergétique et protidique lquantitative et qualitative) sur la gestation et la lactation de la ratte. C. R. Acad. Sci. Paris, 254, 11551157.

CHILDS M. T., TOLLEFSON J., KNOPP R. H., BOWDEN D. A., 1981. Lipid metabolism in pregnancy. VIII. Effects of dietary fat versus carbohydrate on lipoprotein and hepatic lipids and tissue triglyceride lipases. Metabolism, 30, 27-35.

FLINT D. J., SINNET-SMITH P. A., CLEGG R. A., VERNON R. G., 1979. Role of insulin receptors in the changing metabolism of adipose tissue during pregnancy and lactation in the rat. Biochem. J., 182, 421-427.

FOLCH J., LEES M., SLOANE-STANLEY G. H., 1956. A simple method for the isolation and purification of total lipids from animal tissues. J. biol. Chem., 226, 497-509.

HEAP F. C., LODGE G. A., 1967. Changes in body composition of the sow during pregnancy. Anim. Prod., 9, 237-245.

HERRERA E., KNOPP R. H., 1972. Pentose monophosphate shunt dehydrogenases and fatty acid synthesis in late rat pregnancy. Experientia, 28, 646-647.

HITIER Y., CHAMPIGNY O., HOMAYOON P., BOURDEL G., 1982. Circadian feeding pattern in pregnant rats fed two levels of protein. Ann. Nutr. Metab., 26, 129-137.

HUMPHREY J. L., CHILDS M. T., MONTES A., KNOPP R. H., 1980. Lipid metabolism in pregnancy. VII Kinetics of chylomicron triglyceride removal in fed pregnant rat. Am. J. Physiol., 239, E81-E87.

KNOPP R. H., BOROUSH M. A., O'SULLIVAN J. B., 1975. Lipid metabolism in pregnancy. II. Post heparin lipolytic activity and hypertriglyceridemia in the pregnant rat. Metabolism, 24, 481-487.

KNOPP R. H., SAUDEK C. D., ARKY R. A., O'SULLIVAN J. B., 1973. Two phases of adipose tissue metabolism in pregnancy : maternal adaptation for foetal growth. Endocrinology, 92, 984-988.

NAISMITH D. J., RICHARDSON D. P., PRITCHARD A. E., 1982. The utilization of protein and energy during lactation in the rat, with particular regard to the use of fat accumulated in pregnancy. Brit. J. Nutr., 48, 433-441.

PERISSE J., SALMON-LEGAGNEUR E., 1960. Influence du niveau nutritionnel au cours de la gestation et de la lactation sur la production laitière de la ratte. Arch. Sci physiol., 14, 105129.

REMESAR X., AROLA L., PALOU A., ALEMANY M., 1981. Body and organ size and composition during the breeding cycle of rats (Rattus norvegicus). Lab. anim. Sci., 31, 67-70.

SALMON-LEGAGNEUR E., JACQUOT R., 1961. Modifications corporelles entraînées par l'anabolisme gravidique chez la truie. C. R. Acad. Sci., Paris, 253, 544-546. 
SALMON-LEGAGNEUR E., PERISSE J., JACQUOT R., 1960. Relations nutritionnelles entre la gestation et la lactation. C. R. Acad. Sci. Paris, 250, 1921-1923.

SPRAY C. M., 1950. A study of some aspects of reproduction by means of chemical analysis. Brit. J. Nutr., 4, 354-360.

STEINGRIMSDOTTIR L., GREENWOOD M. R. C., BRASEL J. A., 1980. Effect of pregnancy, lactation and a high-fat diet on adipose tissue in Osborne-Mendel rats. J. Nutr., 110, 600-609.

WASFI I., WEINSTEIN I., HEIMBERG M., 1980. Hepatic metabolism of $1-{ }^{14} \mathrm{C}$-oleate in pregnancy. Biochem. biophys. Acta., 619, 471-481. 